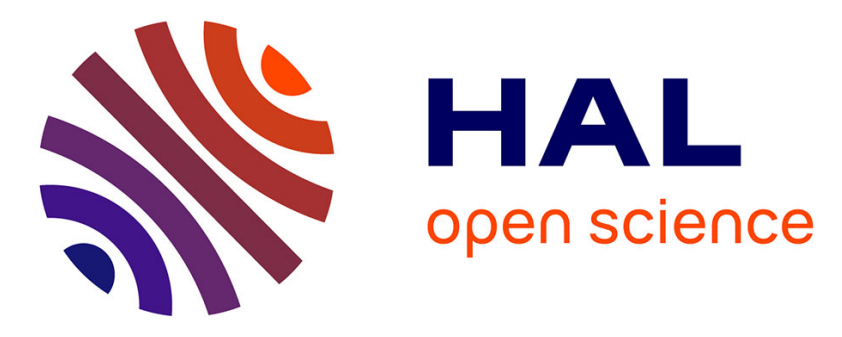

\title{
Measuring shell resonances of spherical acoustic resonators
}

\author{
D. Truong, F. Sparasci, Emmanuel Foltete, Morvan Ouisse, Laurent E Pitre
}

\section{To cite this version:}

D. Truong, F. Sparasci, Emmanuel Foltete, Morvan Ouisse, Laurent E Pitre. Measuring shell resonances of spherical acoustic resonators. International Journal of Thermophysics, 2011, 32 (1-2), pp.427-440. 10.1007/s10765-010-0846-1 . hal-00623001

\section{HAL Id: hal-00623001 \\ https://hal.science/hal-00623001}

Submitted on 23 Mar 2018

HAL is a multi-disciplinary open access archive for the deposit and dissemination of scientific research documents, whether they are published or not. The documents may come from teaching and research institutions in France or abroad, or from public or private research centers.
L'archive ouverte pluridisciplinaire HAL, est destinée au dépôt et à la diffusion de documents scientifiques de niveau recherche, publiés ou non, émanant des établissements d'enseignement et de recherche français ou étrangers, des laboratoires publics ou privés. 
This document is the author's final manuscript of

D. Truong, F. Sparasci, E. Foltête, M. Ouisse and L. Pitre: Measuring shell resonances of spherical acoustic resonators. International Journal of Thermophysics, 32:427-440, 2011.

This paper has been published by Springer and can be found at http://dx.doi.org/10.1007/s10765-010-0846-1 


\title{
MEASURING SHELL RESONANCES OF SPHERICAL ACOUSTIC RESONATORS
}

\author{
D. Truong ${ }^{1,3}$, F. Sparasci ${ }^{1}$, E. Foltête ${ }^{2}$, M. Ouisse ${ }^{2}$, L. Pitre ${ }^{1}$ \\ ${ }^{1}$ Laboratoire Commun de Métrologie LNE-CNAM, Saint-Denis, France \\ ${ }^{2}$ FEMTO-ST Institute, Department of Applied Mechanics, Besançon, France \\ ${ }^{3}$ Corresponding author: daniel.truong@cnam.fr
}

\begin{abstract}
Coupling between gas and shell is a concern in the experiment used at LNE-CNAM to determine the Boltzmann constant $k_{B}$ by an acoustic method. As the walls of real resonators are not perfectly rigid, some perturbations occur in the frequency range of the acoustic resonances measured within helium gas contained in the cavity.

As a contribution for a better understanding of this phenomenon, we have built an experiment to measure the shell modes of the spherical resonators in use in our laboratory. We report here a work in progress to assess these modes using a hammer blow method together with modal analysis. The study is carried out with an air-filled, copper-walled, half-litre quasi-spherical resonator in the frequency range from $1 \mathrm{~Hz}$ to $20 \mathrm{kHz}$.

Our results show that the shell modes expand into multiple resonances of similar modal shape, including the "breathing" mode. We confirm the observations reported in other works $[4,6]$ of shell perturbations at other frequencies than the breathing frequency.
\end{abstract}




\section{Introduction}

There are several methods to determine the Boltzmann constant. One of them is an acoustic method [1] whereby acoustic resonances are measured within a gas contained in a copper quasispherical resonator [2]. Here, the resonant frequencies of the shell are a concern because they can perturb the acoustic modes of the gas in the cavity. When there is coupling between an acoustic mode and a shell mode, the acoustic resonance shifts in frequency, decreases in amplitude and becomes broader. In the LNE-CNAM acoustic experiment used to determine the Boltzmann constant, we detect perturbed acoustic modes by checking the excess half-width of the resonance. In practice, when we encounter an acoustic mode with an unexplained excess halfwidth, we exclude it from the set used to determine the Boltzmann constant.

The mechanism through which motion of the shell causes broadening of the acoustic resonances is still under discussion. Considering only the experimental work, measurements have been made at NIST and at INRiM. One technique consists of exciting the shell with a piezo-transducer (PZT) and measuring the shell response with a phonograph needle or another PZT $[1,3]$. The other technique consists of recording the excess half-widths of a number of acoustic modes while scanning their frequencies, either by changing the temperature [1], as we illustrate below, or by changing the gas-mixture [4]. Resonant frequencies are obtained from the first method and direct coupling effect from the second.

The model used to determine the corrections to experimental resonance frequencies due to shell perturbation [5] predicts a coupling between radial acoustic modes and radial shell motion: the so-called "breathing" mode. But, as shown in the next section $\$ 1.1$, unexplained excess halfwidths of acoustic resonances are not solely observed in the vicinity of the "breathing" frequency. Real resonators have a rather complex geometry compared to that used in the model. To show these differences, a comparison is made in $\$ 1.2$ between measured and calculated resonant frequencies for a hemisphere.

\subsection{Notes on the experimental equipment}

Three different quasi-spherical resonators are used in this study. These resonators are respectively: BCU2v2, BCU1, and LCU. All are half-litre cavities of approximately $10 \mathrm{~mm}$ wall thickness and made of copper (BCU series means Boltzmann and copper). The shapes of these resonators are briefly described in Fig. 1 and their distinctive features are recalled in the respective sections.

The present work will focus on the resonator named LCU for reasons given later in section $\$ 2$. LCU cannot be disassembled as we do not want to alter the alignment of the hemispheres, and hence change the inner shape. So, we have used the north hemisphere of BCU1, whose shape is similar to that of LCU, for the separate hemisphere study described in $\S 1.3$. BCU2v2, which is working in one of our cryostats as an acoustic thermometer, was used to detect shell perturbations through acoustic measurements, which is expanded in section $\$ 1.2$. The results obtained with BCU1 and BCU2v2 are presented in the following sections, to explain the reasons that justified the in-depth investigation of LCU presented in section $\S 2$. 

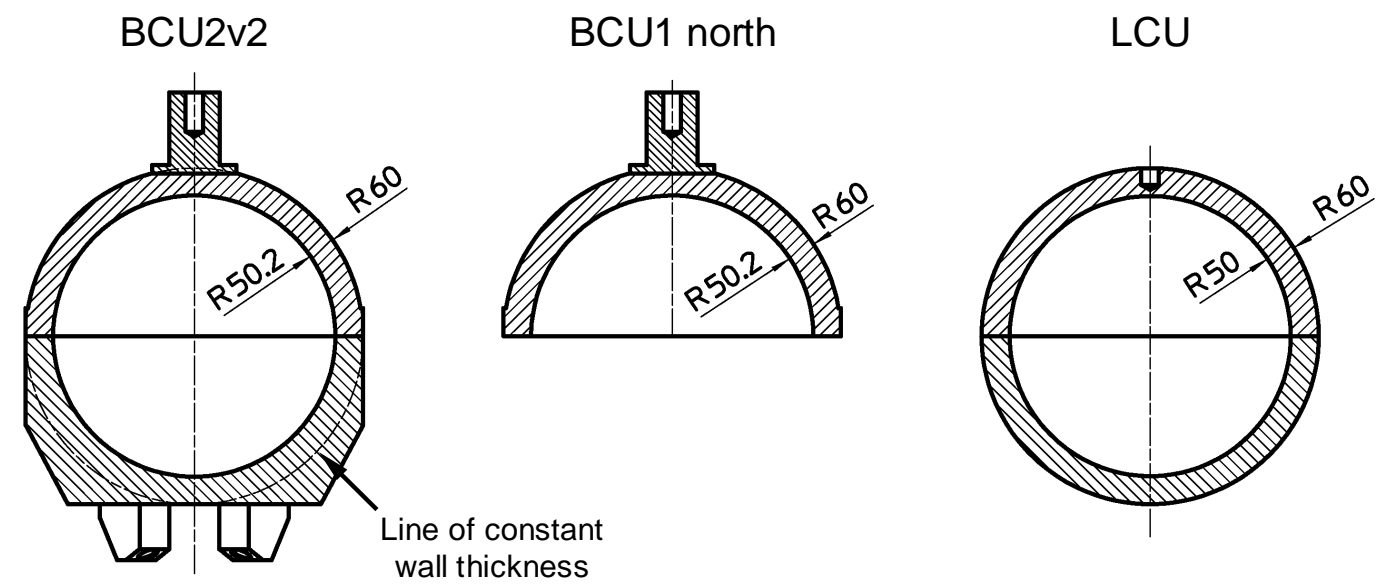

Fig. 1. Sketch of the three resonators used in this work: BCU2v2, BCU1 and LCU. The inner shape is a tri-axial ellipsoid so, the inner radius is the mean value. To make clear to what extent the external shape of BCU2v2 differs from that of a perfect sphere, a line of constant outer radius is overlapped on the drawing. Only the north hemisphere of BCU1 was used for the hemisphere study in $§ 1.2$.

\subsection{Perturbations occur away from the "breathing" mode}

In a recent experiment, we have made a frequency scan of acoustic radial modes by gradually changing the temperature of the spherical resonator BCU2v2. The measurement was conducted under constant gas pressure, since Equation (5) in \$2.4 of Pitre et al [3] shows that shell perturbation is pressure dependant. The sketch of BCU2v2 in Fig.1 shows that this resonator has a shape which differs significantly from a homogeneous sphere in its southern part.

The plot in Fig. 2 shows the observed half-widths, while the excess half-widths is plotted in Fig. 3, of several acoustic resonances as the temperature was lowered from $273 \mathrm{~K}$ to $77 \mathrm{~K}$. The test was carried out with helium gas at constant pressure $p=0.6 \mathrm{MPa}$. According to the mechanical characteristics of BCU2v2, the "breathing" mode of the shell is expected to lie around $19,16 \mathrm{kHz}$ according to the calculation based on the thin-spherical-shell model [5]. But at this frequency, where the "breathing" mode of the sphere is supposed to interfere the most with radial modes within the gas, no excess half-width occurs, while some peaks appear at other frequencies.

An outstanding result is evidenced by this experiment: the perturbations are not temperature dependant. At $13.7 \mathrm{kHz}$ for instance, the $(0,2)$ and $(0,3)$ modes show excess half-widths of several hundred parts in $10^{6}$. At this frequency, the temperature for the $(0,2)$ mode is $273 \mathrm{~K}$ while, for the same frequency, the $(0,3)$ mode is at $77 \mathrm{~K}$. 

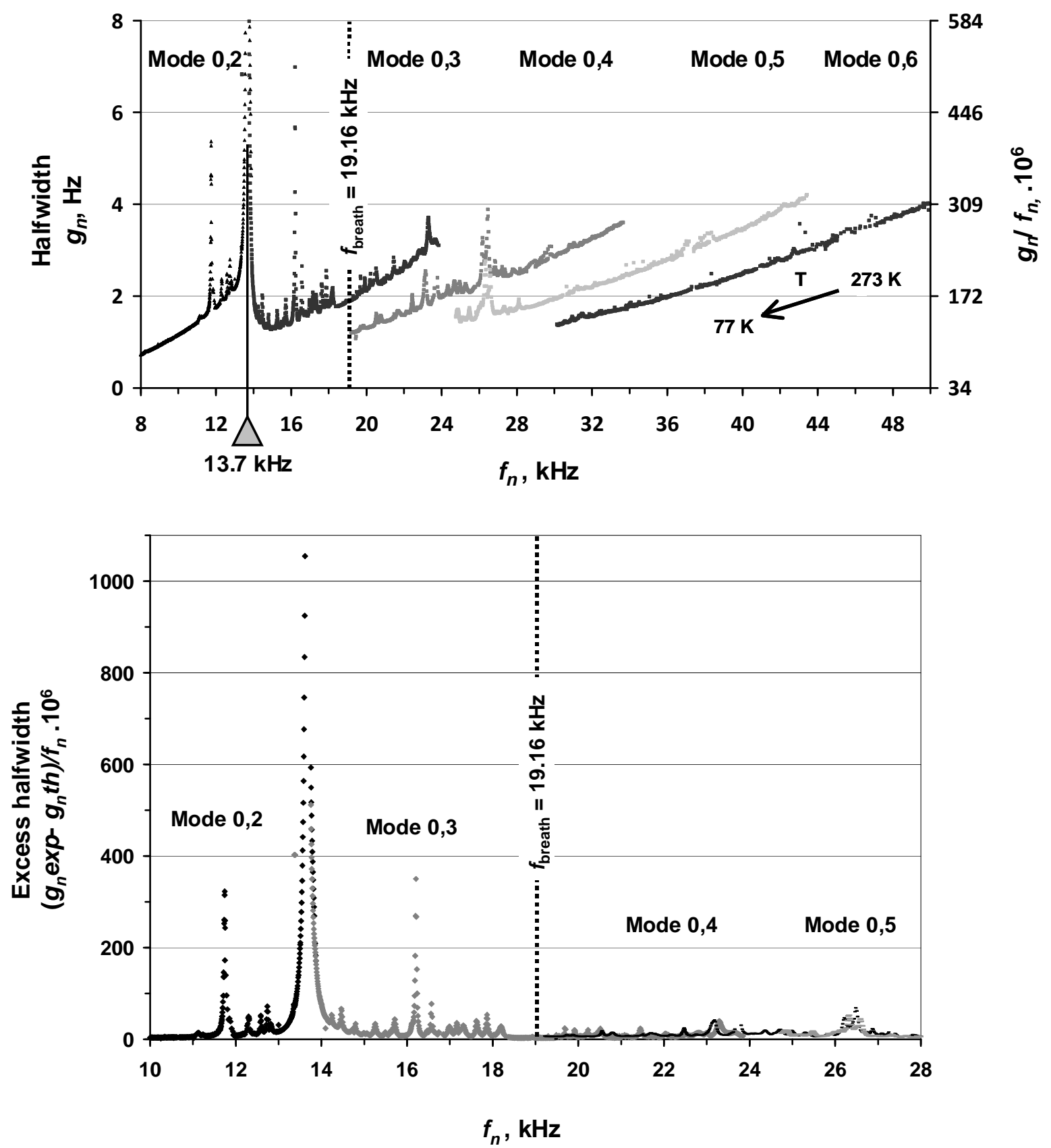

Fig. 2 and Fig. 3. Half-widths and excess half-widths of the acoustic modes as the temperature of the half-litre sphere BCU2v2 was lowered from $273 \mathrm{~K}$ to $77 \mathrm{~K}$. The test was carried out with helium gas at constant pressure $p=0.6 \mathrm{MPa}$. The theoretical breathing frequency of $19.16 \mathrm{kHz}$ is tagged with a dotted line. The grey arrows show how the reduction in temperature sweeps the frequency of each mode. On Fig. 2, the particular frequency of $13.7 \mathrm{kHz}$ is tagged with a triangle for discussion in section $\S 1.2$. 
A comparison was made between calculated and measured resonant frequencies of a half-litre copper hemisphere. A hemisphere was preferred because the effect of the seam between the two halves of an assembled spherical resonator is hard to model. We have a theoretical model for calculating the resonant frequencies of one hemisphere of LCU [5] [7]. Since we are reluctant to disassemble LCU, we have used the north hemisphere of resonator BCU1 whose shape is similar to that of LCU. As shown on the left-hand drawing of Fig. 4, the exterior of LCU is perfectly spherical, while BCU1 has a cylindrical support screwed on the top.

The resonant frequencies of the hemisphere were calculated using the theoretical model. Then, the response of the north hemisphere of BCU1 to a hammer blow was measured by a piezoaccelerometer glued onto the outer surface. The experimental method is described in detail in section §2. Only one spectrum was recorded at a single position of the accelerometer and the impact. As shown in Fig. 4 a lack of agreement has been found between our experimental determination of the resonant frequencies and these calculations.

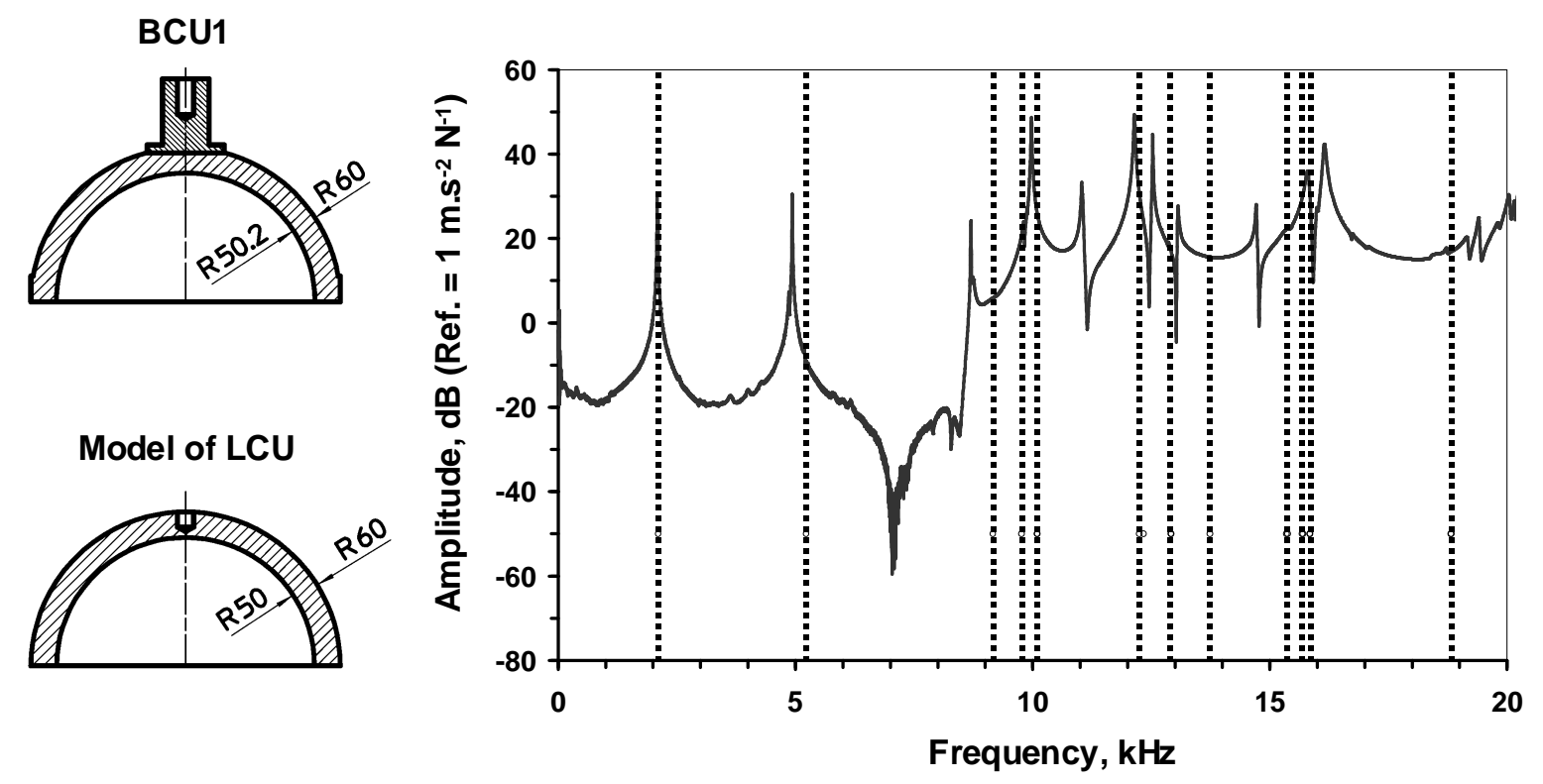

Fig. 4. Comparison between experimental spectrum and resonant frequencies calculated by Mehl [7] for a half-litre copper hemisphere. The experimental data plotted using a solid line is the response of BCU1 north hemisphere to a hammer blow. The resonant frequencies calculated for the north hemisphere of LCU are shown using black dotted lines. The two hemispheres differ slightly, as shown on the left-hand drawings.

\subsection{Objectives}

On account of the differences found between experimental data and the theory, particularly as the two agree well together for low-frequency modes, it was deemed necessary to further 
investigate the natural modes of vibration of the shell experimentally. The objectives of this work were to provide experimental data for comparison with the observed excess half-widths of the acoustic modes, and to provide suitable data for the validation of future theoretical models.

\section{Method and Results}

\subsection{Method}

Our method is divided into several stages that we will expand in the following sections. First, we collect a variety of data sets of the response of the shell to impacts. Then we build Frequency Response Functions (FRFs) from both the response of the accelerometer and the impact of the hammer of the shell. Finally, we apply modal analysis techniques to the FRF set to obtain estimates of the mode parameters and display the modal shape.

\subsection{Measurement of the shell response}

The experiment consists of exciting the shell modes of a quasi-spherical resonator with a calibrated impact hammer. The resonator is at room temperature and filled with air. We measure the response of the shell using three accelerometers glued onto the surface. The frequency range of the accelerometers is $0-20 \mathrm{kHz}$. A FRF is built from 24000 samples recorded at the rate of $120 \mathrm{kS} / \mathrm{s}$. The location of these accelerometers is shown in Fig. 4. This ring-down method was chosen because it is easy to implement, and therefore a large number of data sets, with impacts at different locations, can be acquired. Many Frequency Response Functions (FRF) are required to achieve an accurate modal analysis. We define these functions as

$$
F R F_{i j}=\frac{A_{i}(\omega)}{F_{j}(\omega)}
$$

Where $A_{i}(\omega)$ is the Fast Fourier Transform (FFT) of $a_{i}(t)$, the signal from accelerometer $i$, and $F_{j}(\omega)$ the FFT of $f_{i}(t)$, the impact signal $j$.

The quasi-spherical resonator chosen for this experiment is the LCU copper sphere. Since the LCU spectrum shows relatively few peaks that are well separated in frequency, this sphere is a good tool for a first experiment. The external shape of this particular resonator lies very close to that of a perfect sphere, with an inner diameter of $100 \mathrm{~mm}$ and a wall thickness of $10 \mathrm{~mm}$. The spherical symmetry is broken only by the two thermometer housings that lie on the outer surface and the screw at the top.

The sphere was suspended from a wire in a freely-hanging configuration, as shown in Fig. 6. Impacts were made at 30 different locations all over the surface of the sphere (Fig. 5). The locations are optimized to excite all modes with the highest amplitude. 


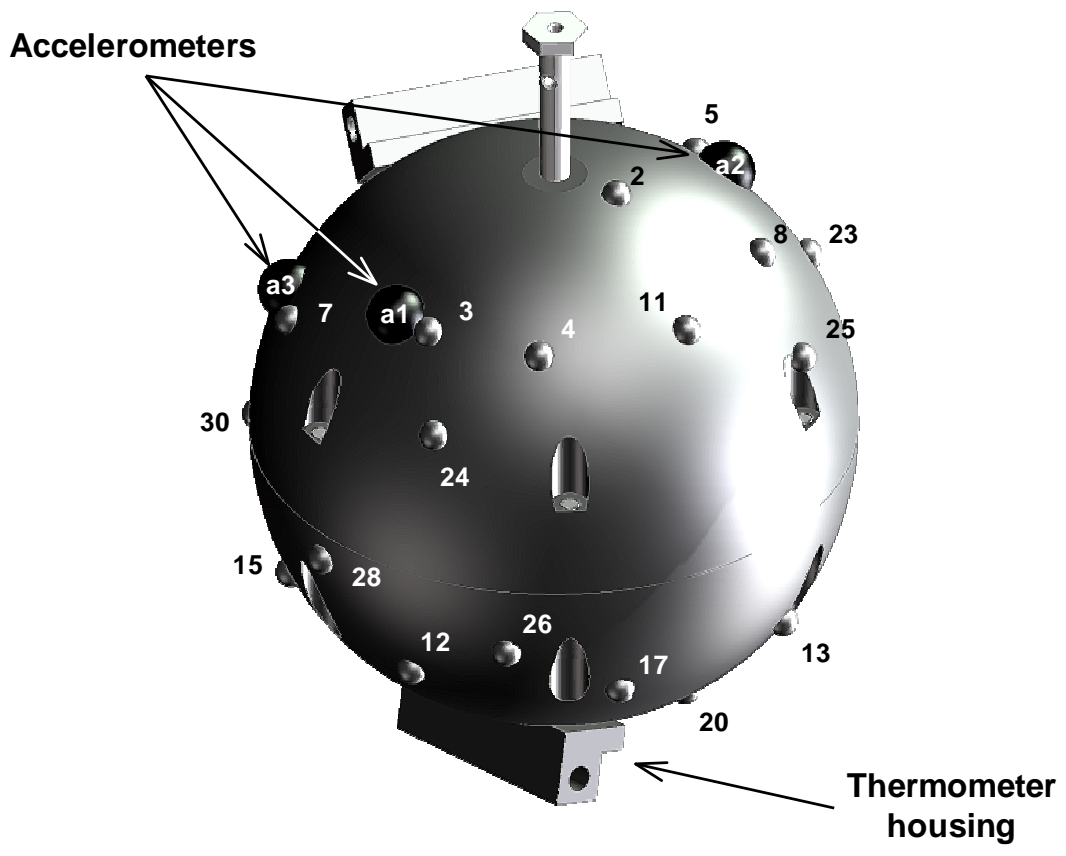

Fig. 5. A 3D view of the impact locations on LCU. The big dots $a_{i}$ are the locations of the accelerometers. The small dots are the impact locations.

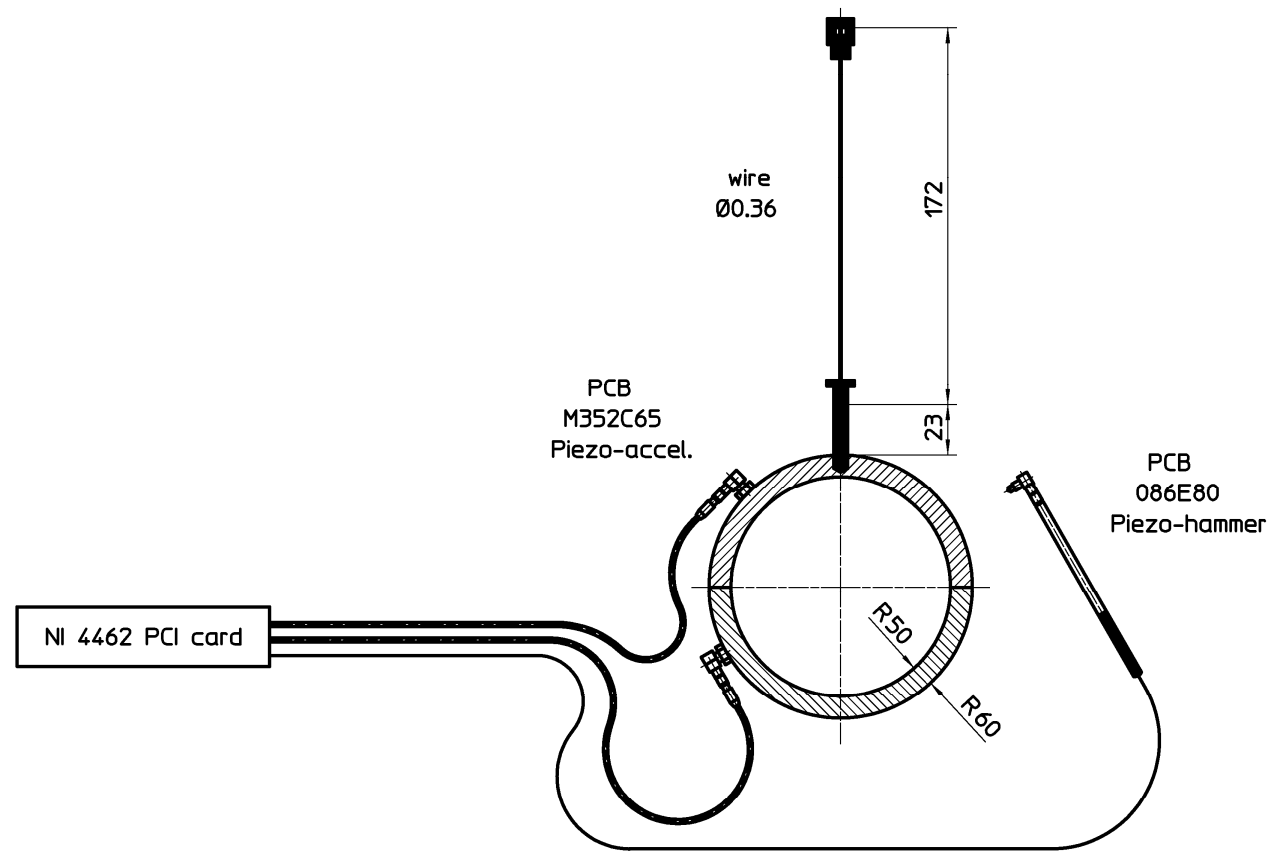

Fig. 6. Sketch of the experimental set-up. Sphere LCU is suspended from a steel wire. The three accelerometers (only two are shown) are glued onto the sphere. 
The FRFs obtained for all impacts as well as the coordinates of the impacts were entered into a modal analysis software package called MODAN. This software package was developed by one of us (E. F.) [8]. Fig. 7 shows the response recorded by three accelerometers located at different positions on the shell to the same impact. This figure makes it clear that the response is different in magnitude, and to a lesser extent in frequency, when a different location on the sphere is chosen. Moldover et al. observed a similar effect with their phonograph needle experiment [1].



Fig. 7. Response of the shell to the same impact recorded from three different positions. It is a sub-set from the 30 FRFs. Each curve is the plot of the FRF recorded by one of the three accelerometers glued onto the sphere. The vertical dotted line shows the frequency of the "breathing" mode of highest amplitude at $18.7 \mathrm{kHz}$. The grey line shows an antiresonance at 4 $\mathrm{kHz}$ caused by the vicinity of the impact to the accelerometer recording this FRF.

\subsection{Modal analysis}

Several methods are available for modal analysis. We have used a Least Squares Complex method in Frequency domain (LSCF) to detect modes from all the FRFs, synthesised in a Complex Mode Indicator Function. The mode identification is made manually by choosing the best fitting modes from a graphical representation, and is therefore subject to fluctuations. Indeed, the frequency can shift by as much as $10 \mathrm{~Hz}$ from one identification to another. When the process is completed, the software gives estimates of modal parameters: frequency, damping and modal shape. The results from one modal analysis of LCU are tabulated in Appendix 1.

The three pictures shown in Fig. 8 are an example of three sequential frames taken from a video generated by the MODAN software. The video shows the motion of the shell generated by a 
shell mode at 7,9 kHz. Each closed line represents an ellipse connecting points at the same latitude on the shell. We can see that this particular mode "squeezes" the sphere on an axis close to the vertical. The left-hand frame shows the two poles "stretching" the sphere while the righthand frame shows them "squeezing" the sphere.
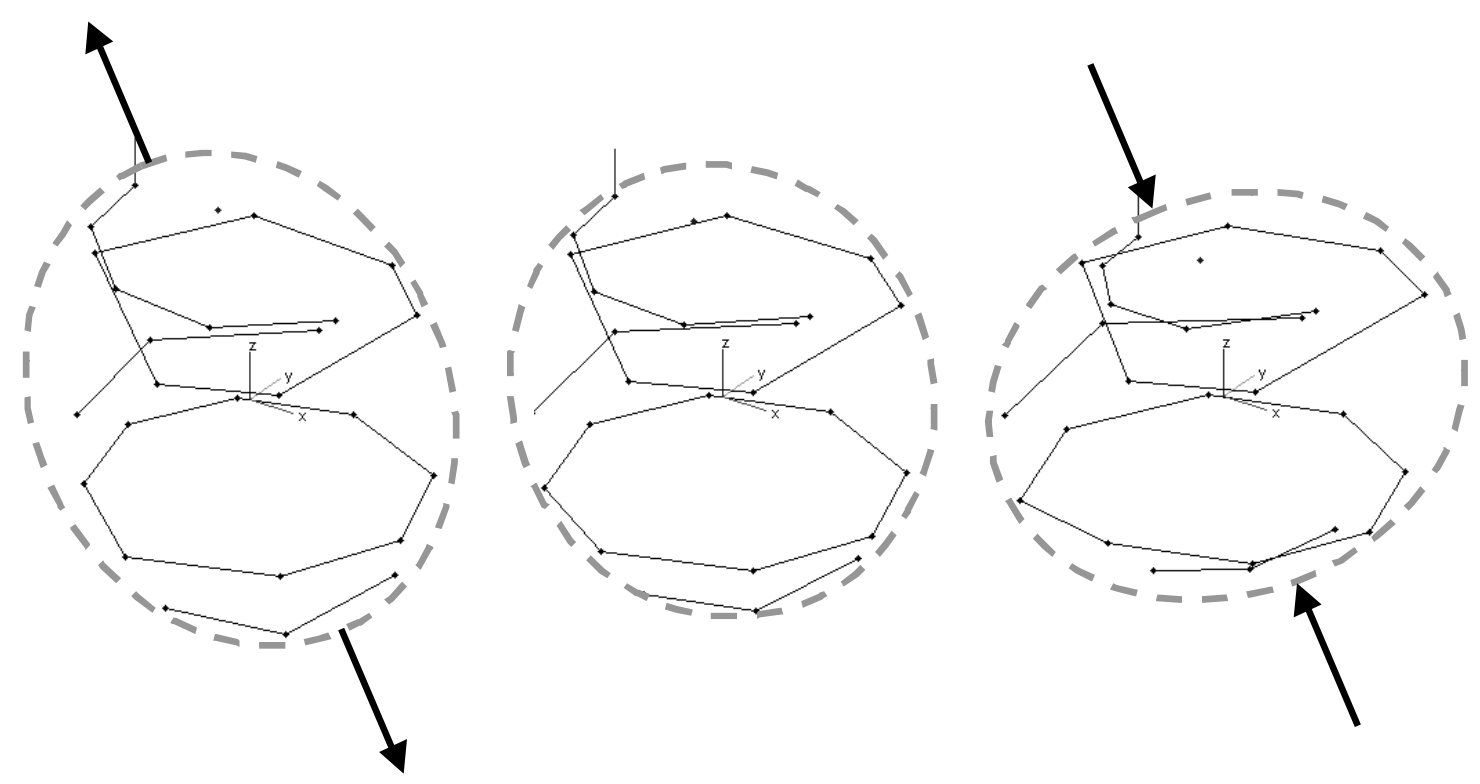

Fig. 8. Display of modal shape with MODAN. The points show the location of impacts. The segments are drawn between points of approximately equal latitude. The modal shape function plays a video showing the displacement of the points. Shown here is a series of views for the $7,9 \mathrm{kHz}$ LCU shell mode. The arrows highlight the compression and extension of the sphere along a near-vertical axis.

\subsection{Results}

With the hammer blow technique together with modal analysis we have assessed the shell modes in the frequency range $1 \mathrm{~Hz}$ to $20 \mathrm{kHz}$ for LCU. The observation of the modal shapes all along the spectrum provides an idea of a topology for the modes, depending on the frequency. Three frequency ranges have been identified, and are shown in Fig. 9. The first modes to appear are "squeezing" modes at frequencies below $10.4 \mathrm{kHz}$, then "shaking" modes from $10.4 \mathrm{kHz}$ to 18.2 $\mathrm{kHz}$, and finally "breathing" modes from $18.2 \mathrm{kHz}$ to $20 \mathrm{kHz}$. For frequencies above $20 \mathrm{kHz}$, the modes are more difficult to describe; moreover their frequencies lie beyond the range of the accelerometer employed. Some calculation were made using finite element NASTRAN software package as a help to identify visually the modes from MODAN modal shape displays. Fig. 10 shows the modal shape calculated with this software package for typical "squeezing", "shaking" and "breathing" modes. A "squeezing" mode is a mode showing two poles stretching or squeezing the sphere on one axis. A "shaking mode" is a mode where the deformation is more complex with a bending or a torsion effect on the poles. A "breathing" mode is an almost isotropic mode showing the surface expanding or contracting uniformly. 


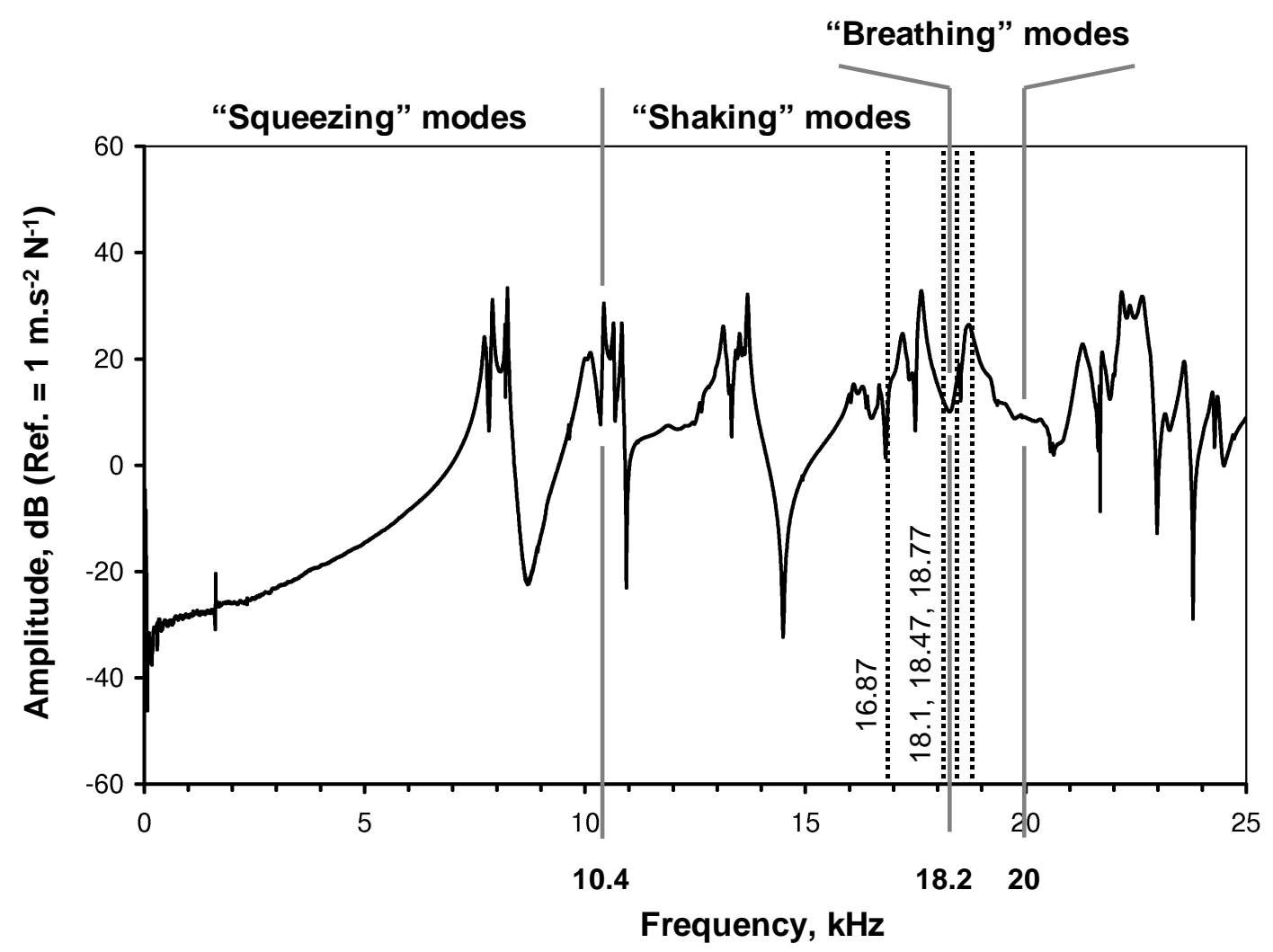

Fig. 9. Topology of the modes for the LCU sphere. The background plot is a typical LCU spectrum. Boundaries have been set after observation of all deformation shapes provided by MODAN. The frequencies of observed acoustic perturbations, reported in [3], have been tagged by dotted lines at $16.87 \mathrm{kHz}, 18.10 \mathrm{kHz}, 18.47 \mathrm{kHz}$ and $18.77 \mathrm{kHz}$.

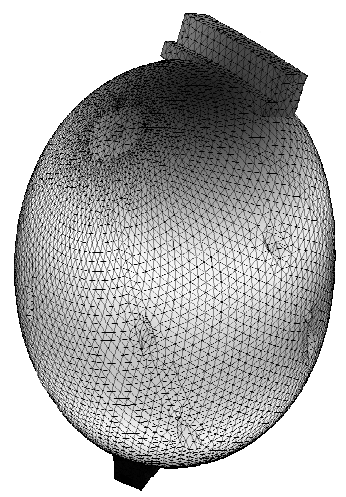

"Squeezing" mode

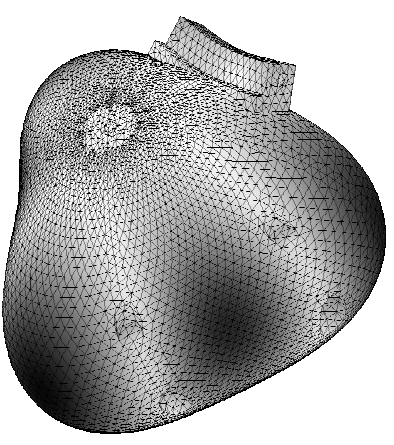

"Shaking" mode

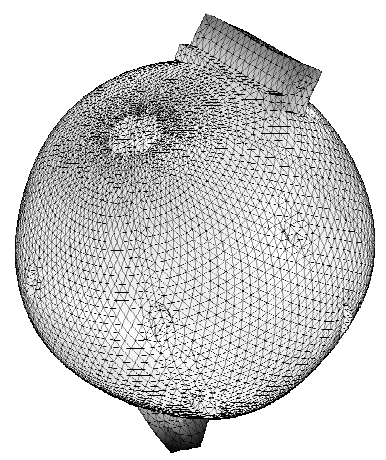

"Breathing" mode

Fig. 10. Illustration of typical deformation shapes. The deformation shapes for LCU sphere are calculated using the software package NASTRAN [9]. These displays are dimensionless. The darker is the area, the higher is the relative displacement. 
Jim Mehl gives an alternate classification of the shell modes in [5], together with a plot of the shell resonance eigenvalues $\Lambda_{\mathrm{ns}}$ as a function of the ratio between the inner radius $a$ and the outer radius $b$. This plot is reproduced in Fig. 11 and the ratio for LCU, $b / a=1.2$, is tagged with a solid line. We give the correspondence between our "squeezing", "shaking" and "breathing" modes and the "bending" and "extensional" categories described in [5].

\section{Higher frequencies}

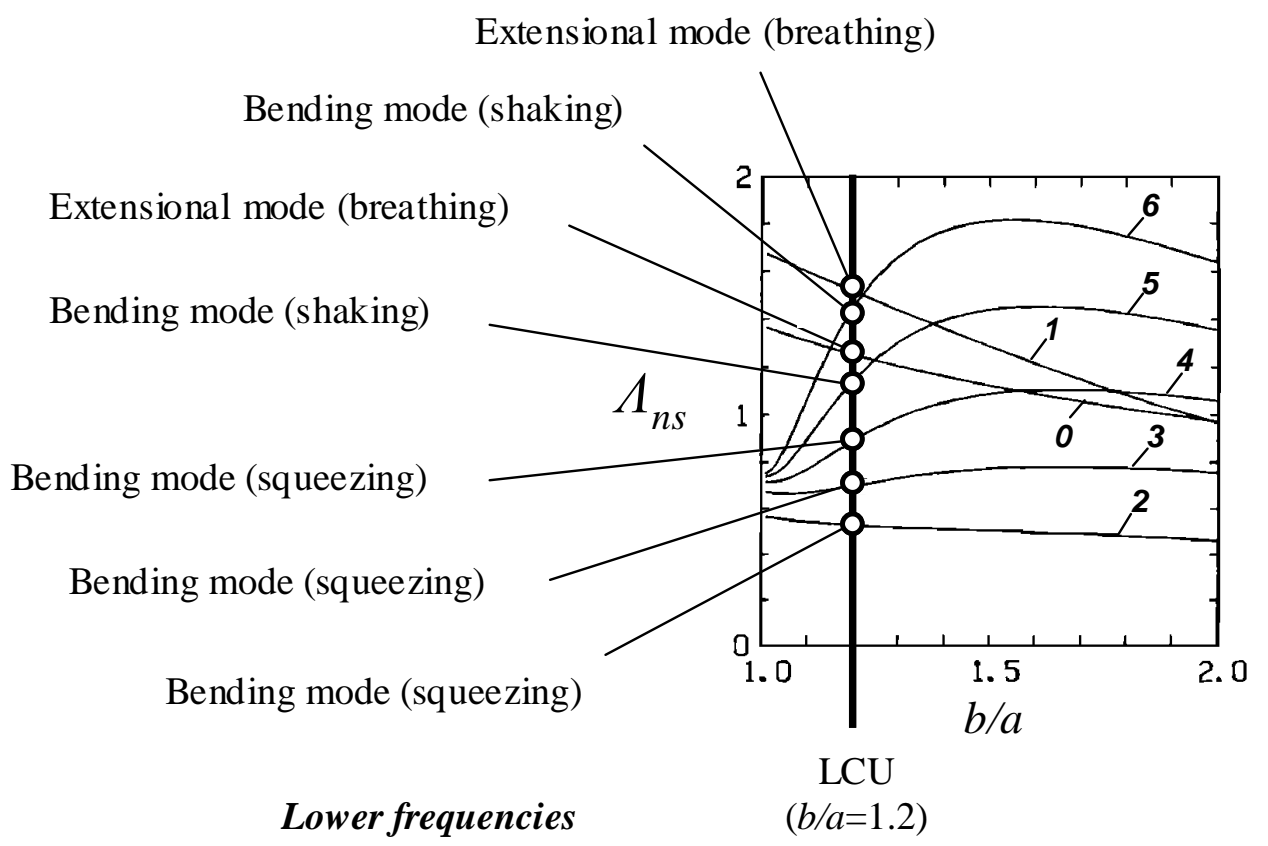

Fig. 11. Correspondence between our "squeezing", "shaking" and "breathing" modes and the classification found in [5]. The background plot is a reproduction of the original fig. 1 contained in this paper, where the resonance frequencies $\Lambda_{\mathrm{ns}}$ are plotted as a function of $b / a$. The curves are labeled with the mode index $\mathrm{n}$. The intersections between the $b / a$ ratio for LCU and the curves give the theoretical mode series from lower to higher frequencies.

Now, we compare the frequencies of the shell modes given for LCU by Pitre et al. in [3] to the modes identified with our method. The theoretical "breathing" frequency $\Lambda_{01}$ calculated from the thin-shell model developed by Jim Mehl in [5] is $19 \mathrm{kHz}$. The experimental "breathing" frequency $f_{\text {breath }}$ resulting from a fit to acoustic slopes is $17.42 \mathrm{kHz}$ [3]. The formulas used to calculate these frequencies are recalled below:

$\Lambda_{01}$ is the root of:

$$
\left[\left(q A^{2}-1\right)\left(q B^{2}-1\right)+A B\right]-(1+q A B)(B-A)=\text { zero }
$$


Where:

$$
\begin{aligned}
& q=\frac{1}{2}(1-\sigma) /(1-2 \sigma) \\
& A=k_{l} a, B=k_{l} b, \text { and } \\
& k_{l}=\frac{2 \pi f}{[E(1-\sigma) / \rho(1-2 \sigma)(1+\sigma)]^{1 / 2}}
\end{aligned}
$$

Where $a=50 \mathrm{~mm}$ is the inner radius, $b=60 \mathrm{~mm}$ is the outer radius, $E=122.9 \mathrm{GPa}$ is the Young's modulus, $\sigma=0.355$ is the Poisson coefficient, and $\rho=8900 \mathrm{~kg} / \mathrm{m}^{3}$ is the density.

The value of $f_{\text {breath }}$ is found experimentally, from the best-fit to the speed-of-sound data from a number of acoustic modes [3]. The formula that describes the effect of the shell breathing mode on the frequency of a radial acoustic mode is:

$$
\begin{aligned}
& \frac{\Delta f_{\text {elastic }}}{f_{(0, n)}} \approx \frac{\kappa p}{1-\left(f_{(0, n)} / f_{\text {breath }}\right)^{2}} \\
& \kappa=\frac{5 a}{6 t \rho u_{\text {shell }}^{2}}
\end{aligned}
$$

Where $t$ is the thickness of the shell and $u_{\text {shell }}$ is the longitudinal speed of sound in the shell.

If there is a good agreement between the theoretical "breathing" frequency and our range of "breathing" modes, on the other hand we are unable to explain the experimental "breathing" frequency found in such paper. Nevertheless, Pitre et al. have pointed out that - when this experimental frequency is applied to correct acoustic data - very consistent results are found with different gases for all acoustic modes. 


\section{Conclusion}

This study shows that shell modes expand into multiple resonances of similar modal shape. The geometry of real resonators is more complex than that used to build theoretical models. A real resonator has holes for the screws, housings to accommodate thermometers, plugs that contain transducers etc. This non-homogeneous shell lifts degeneracy and the result is a series of modes of similar shape. An interesting result is that the "breathing" mode also is affected. We have observed a series of similar "breathing" modes over a relatively large frequency range of $2 \mathrm{kHz}$, centred around $18.7 \mathrm{kHz}$. Therefore, to consider the "breathing" mode as a singular mode appearing at one sharp frequency is no longer possible for real resonators.

Compared to previous results with the same sphere [3], that the perturbed frequencies measured at $16.87 \mathrm{kHz}$ and $18.10 \mathrm{kHz}$, are in the range of non-radial modes of the shell, while those recorded at $18.47 \mathrm{kHz}$ and $18.77 \mathrm{kHz}$ are in the range of "breathing" modes. With a main breathing mode measured at $18.7 \mathrm{kHz}$, we find a good agreement with the theoretical breathing frequency $\Lambda_{01}=19 \mathrm{kHz}$ calculated with [5].

To summarise, we have developed a hammer blow technique with which we can directly measure the frequencies of the shell modes of real, quasi-spherical resonators. By using modal analysis techniques we are able to assess the damping factors and deformation shapes of these modes. What we still cannot assess is the amplitude of force or displacement of a single mode. This is due to the ring-down technique employed, with which the energy put into the system is dissipated into many shell modes. In order to find the displacement of a given point on the surface associated to one mode, one has first to determine the contribution of each mode and, for one particular mode, how the force is distributed all over the shell.

The technique has scope for improvement. In particular, the number of impacts must be increased for future measurements because the exploitation of data is mainly visual. In the current experiment, in which 30 impacts were measured, it was almost impossible to distinguish between multi-poles modes. Therefore, no complete comparison between the modes calculated with NASTRAN and those obtained with MODAN could be made. Moreover, a more accurate identification of the modes would be achievable by testing different modal analysis methods and selecting the best results, but this is very time-consuming. 


\section{Acknowledgements}

The authors acknowledge J. B. Mehl whose work stimulated this study. They have received valuable support from J.-L. Le Carrou from LAM-Université Pierre \& Marie Curie- Paris VI (France), who checked and prepared the data prior to analysis using the MODAN software. D.T. is indebted to P. A. Giuliano Albo from INRiM, Turin (Italy), for providing notes about gas and shell coupling [10] and as well as constant comments about the work. He gratefully acknowledges P. Gélat from NPL, Teddington (UK), for helpful discussions and R. Gavioso from INRiM, Turin (Italy), for sharing unpublished results. We thank Mark Plimmer, from LNECNAM, Saint-Denis (France) and Robin Underwood from NPL, Teddington (UK) for carefully reading the manuscript.

\section{References}

[1] M. R. Moldover, J. P. M. Trusler, T. J. Edwards, J. B. Mehl, R. S. Davis: Measurement of the Universal Gas Constant R using a spherical acoustic resonator, Journal of research of the National Bureau of Standards, 93(2), 85-144, (1988)

[2] L. Pitre, C. Guianvarc'h, F. Sparasci, A. Richard and D. Truong: Progress towards an Acoustic/Microwave Determination of the Boltzmann Constant at LNE-INM/CNAM, Int. J. Thermophys., 25, 1730-1739, (2008)

[3] L. Pitre, M. Moldover, W. L. Tew: Acoustic thermometry: new results from $273 \mathrm{~K}$ to $77 \mathrm{~K}$ and progress towards 4K, Metrologia, 43, 142-162 (2006)

[4] R. M. Gavioso, G. Benedetto, R. Cuccaro, C. Guianvarc'h, D. Madonna Ripa, P. A. Giuliano Albo: Shell perturbation of an acoustic thermometer determined from speed of sound in gas mixtures, submitted to Int. J. Thermophys.

[5] J. B. Mehl: Spherical acoustic resonator: effect of shell motion, J. Acoust. Soc. Am., 78(2), 782-788, (1985).

[6] M.B. Ewing and A.R.H. Goodwin: Speeds of sound, perfect-gas heat capacities, and acoustic virial coefficients for methane determined using a spherical resonator at temperatures between $255 \mathrm{~K}$ and $300 \mathrm{~K}$ and pressures in the range $171 \mathrm{kPa}$ to $7.1 \mathrm{Mpa}$, J. Chem. Thermodynamics, 24, 1257-1274, (1992).

[7] J. B. Mehl: LCU shell response 2009-06-28 (unpublished), 15 pages of notes describing finite-element calculations of modes for the LCU hemisphere, from the author to L. P.

[8] E. Foltête, G. M. L. Gladwell and G. Lallement: On the reconstruction of a damped vibrating system from two complex spectra, part 2: experiment, Journal of Sound and Vibration, 240(2), 219-240, (2001)

[9] M. Ouisse: Notes de calcul: préparation de la campagne de mesure LCU 2009-09-15 (report), 14 pages reporting on finite-element calculations of the first 44 modes of LCU sphere using NASTRAN software, from the author to D. T.

[10] P. A. Giuliano Albo: Gas-Shell coupling: shell motion 2009-07-02 (notes), 3 pages of notes describing a simple classification of modes obtained with finite-element calculations using SALOME-MECA software, from the author to D. T. 
Appendix 1 Modal parameters obtained with MODAN for LCU sphere.

The modal parameters of natural frequency calculated from the set of FRFs are tabulated

\begin{tabular}{|c|c|c|c|c|c|c|c|}
\hline $\begin{array}{c}\text { Mode } \\
\mathrm{n}^{\circ}\end{array}$ & $\begin{array}{l}\text { Frequency } \\
(\mathrm{Hz})\end{array}$ & $\begin{array}{c}\text { Damping } \\
\text { factor (\%) }\end{array}$ & $\mathrm{MPC}^{\mathrm{a}}(\%)$ & $\begin{array}{c}\text { Mode } \\
n^{\circ}\end{array}$ & $\begin{array}{l}\text { Frequency } \\
(\mathrm{Hz})\end{array}$ & $\begin{array}{c}\text { Damping } \\
\text { factor }(\%)\end{array}$ & $M C^{a}(\%)$ \\
\hline 1 & 7651.51 & 0.44 & 36.10 & 26 & 13300.34 & 0.20 & 59.56 \\
\hline 2 & 7655.21 & 0.67 & 26.94 & 27 & 13437.60 & 0.11 & 20.34 \\
\hline 3 & 7662.66 & 0.36 & 48.67 & 28 & 13507.76 & 0.09 & 64.11 \\
\hline 4 & 7722.15 & 0.36 & 69.31 & 29 & 13590.92 & 0.14 & 72.39 \\
\hline 5 & 7752.19 & 0.15 & 38.78 & 30 & 13607.58 & 0.10 & 62.75 \\
\hline 6 & 7898.27 & 0.20 & 78.69 & 31 & 13690.30 & 0.11 & 94.47 \\
\hline 7 & 7904.49 & 0.15 & 17.77 & 32 & 16026.11 & 0.12 & 19.11 \\
\hline 8 & 8202.69 & 0.09 & 4.57 & 33 & 16101.18 & 0.44 & 91.07 \\
\hline 9 & 8202.92 & 0.09 & 4.67 & 34 & 16325.61 & 0.58 & 50.16 \\
\hline 10 & 8204.29 & 0.11 & 13.91 & 35 & 16693.21 & 0.19 & 13.74 \\
\hline 11 & 8245.33 & 0.08 & 95.96 & 36 & 16837.15 & 0.46 & 5.89 \\
\hline 12 & 10000.41 & 0.64 & 51.07 & 37 & 16838.44 & 0.44 & 7.41 \\
\hline 13 & 10013.38 & 0.58 & 15.60 & 38 & 16864.39 & 0.21 & 14.94 \\
\hline 14 & 10092.29 & 0.55 & 6.80 & 39 & 17209.33 & 0.37 & 41.31 \\
\hline 15 & 10112.70 & 0.61 & 32.22 & 40 & 17320.54 & 0.12 & 52.58 \\
\hline 16 & 10423.63 & 0.14 & 91.13 & 41 & 17529.74 & 0.23 & 84.00 \\
\hline 17 & 10585.16 & 0.12 & 88.87 & 42 & 17614.15 & 0.27 & 28.37 \\
\hline 18 & 10655.20 & 0.10 & 88.50 & 43 & 17639.12 & 0.20 & 77.94 \\
\hline 19 & 10835.95 & 0.12 & 80.31 & 44 & 18405.42 & 0.42 & 76.16 \\
\hline 20 & 12465.17 & 0.21 & 48.13 & 45 & 18513.09 & 0.04 & 28.81 \\
\hline 21 & 12599.66 & 0.86 & 12.36 & 46 & 18682.74 & 0.23 & 63.92 \\
\hline 22 & 12612.11 & 0.92 & 19.47 & 47 & 18717.32 & 0.19 & 54.86 \\
\hline 23 & 12653.86 & 0.57 & 2.44 & 48 & 19253.22 & 0.35 & 33.93 \\
\hline 24 & 12960.05 & 0.12 & 30.51 & 49 & 20279.10 & 0.40 & 33.42 \\
\hline 25 & 13148.55 & 0.31 & 44.41 & 50 & 21295.24 & 0.35 & 34.57 \\
\hline
\end{tabular}

${ }^{\text {a }}$ The Modal Phase Collinearity (MPC) is an indicator of the accuracy of the mode obtained. The higher it is, the more reliable is the identification. 